María José Magliano

Universidad Nacional de Córdoba, Argentina.

\title{
Interseccionalidad y migraciones: potencialidades y desafíos
}

Resumen: Los debates sobre la interseccionalidad se han vuelto centrales en las elaboraciones teóricas sobre género y migraciones. Este artículo explora las potencialidades de la interseccionalidad para pensar procesos y experiencias sociales, específicamente los procesos migratorios internacionales y las experiencias de los y las migrantes, a la vez que reflexiona acerca de sus puntos grises y 'problemas'. Para ello, historiza el surgimiento y consolidación de la perspectiva interseccional, sus implicancias en el ámbito de las ciencias sociales en general y las migraciones en particular, y también las ambigüedades teóricas y metodológicas que la perspectiva presenta. Por último, el artículo recupera las contribuciones de la interseccionalidad para analizar empíricamente la vinculación entre migración y trabajo, a partir de indagar acerca de trayectorias laborales de mujeres migrantes peruanas en el empleo doméstico remunerado en Argentina. En torno a ello, se nutre de un trabajo de campo cualitativo (entrevistas en profundidad y observación participante) realizado desde el año 2011 hasta la actualidad en la ciudad de Córdoba (Argentina) con mujeres migrantes de origen peruano que se dedican o se dedicaron en algún momento de su experiencia migratoria - a esta actividad laboral. Palabras claves: Interseccionalidad; migración internacional; clasificaciones sociales, estudios de género; trabajo doméstico remunerado.

(c) $(1)$ Esta obra tem licença Creative Commons.

1 Floya ANTHIAS, 2012, p. 102

2 Leslie MCCALL, 2005

\section{Introducción}

Los debates sobre la interseccionalidad se han vuelto centrales en la teorización sobre género y migraciones. ' Dicha perspectiva ha captado la atención de cada vez más investigadoras/es a nivel global y latinoamericano, convirtiéndose en una de las contribuciones teóricas más importantes de los estudios feministas. ${ }^{2}$ En términos generales, la perspectiva interseccional, que emerge como una apuesta teórico-metodológica para comprender las relaciones sociales de poder y los contextos en que se producen las desigualdades sociales, hace posible un análisis 'complejo' de la realidad vivida por los sujetos, mujeres y varones, 
mediante el abordaje de las diferentes posicionalidades y clasificaciones sociales, históricamente situadas. ${ }^{3}$

Este artículo explora las potencialidades de la interseccionalidad para pensar procesos y experiencias sociales, específicamente los procesos migratorios internacionales y las experiencias de los y las migrantes, a la vez que reflexiona acerca de sus puntos grises y 'problemas'. Para ello, en los dos primeros apartados historiza el surgimiento y consolidación de esta perspectiva, sus implicancias en el ámbito de las ciencias sociales en general y las migraciones en particular y también las ambigüedades teóricas y metodológicas que la interseccionalidad presenta, en especial debido a que su explosión como elaboración teórica no estuvo acompañada por investigaciones

${ }^{4}$ La selección de textos realizada para tal análisis no intenta ser exhaustiva ni reconstruir el completo estado de la cuestión del debate sobre interseccionalidad a nivel global y latinoamericano.

${ }^{5}$ Guillermo NEIMANN y Germán QUARANTA, 2007, p. 219. empíricas. ${ }^{4}$ En el tercer apartado recupera las contribuciones de la interseccionalidad para analizar la vinculación entre migración y trabajo, a partir de indagar acerca de trayectorias laborales de mujeres migrantes peruanas en el empleo doméstico remunerado en Argentina. En torno a ello, se nutre de un trabajo de campo cualitativo (entrevistas en profundidad y observación participante) realizado desde el año 2011 hasta la actualidad en la ciudad de Córdoba con mujeres migrantes de origen peruano que se dedican o se dedicaron en algún momento de su experiencia migratoria - a esta actividad laboral. La gran mayoría de las investigaciones enfocadas en estudiar trayectorias laborales migrantes han partido del supuesto de la existencia de una cierta homogeneidad entre quienes se desempeñan en una misma actividad económica. En tensión con esta premisa, la perspectiva interseccional, al reflexionar sobre las posicionalidades múltiples de los sujetos - incluso de aquellos que forman parte de un mismo grupo o sector -, viene a disputar cualquier intento de generalización. En este artículo se esbozan algunas ideas en esta dirección a partir de estudios de caso - que consisten en el abordaje de lo particular priorizando el caso único, donde la efectividad de la particularización reemplaza la validez de la generalización ${ }^{5}$ - de trayectorias laborales de mujeres peruanas (Daniela, Gisella y Raquel) cuya primera inserción en Córdoba fue el trabajo doméstico remunerado.

Si bien la interseccionalidad ha cobrado un creciente interés en los últimos decenios, es escasa la literatura que analice sobre sus potencialidades en el ámbito de los estudios sobre migraciones internacionales. Tal vez porque surgió al calor de las luchas de las feministas negras en Estados Unidos, se ha expandido especialmente por los países del 'norte' y sólo recientemente ha adquirido mayor centralidad en América Latina en su conjunto. Precisamente, este artículo pretende llenar algunos de estos vacíos. 
6 Kimberley CRENSHAW, 1991; Patricia HILL COLLINS, 1993.

7 Irene BROWNE y Joya MISRA, 2003; CRENSHAW, 1991; HILL COLLINS, 1993.
${ }^{8}$ María LUGONES, 2005, p. 62-63.

\section{Tras los caminos de la intersecciona- lidad: una mirada sobre sus orígenes y aportes principales}

Las preocupaciones en torno a la interseccionalidad comienzan a desarrollarse desde los movimientos de feministas negras en Estados Unidos en su intento por deconstruir las categorías 'mujeres' y 'negras'. Su surgimiento se fundó en la necesidad de reflexionar sobre - y encontrar respuestas para - los procesos de producción y reproducción de desigualdades sociales, siendo las mujeres de color en aquel país su primer grupo objeto de estudio. Los trabajos pioneros de Crenshaw y Hill Collins se concentraron en las dimensiones de género y raza para pensar las múltiples desigualdades y las formas de opresión y subordinación que enfrentaban las mujeres negras en Estados Unidos. ${ }^{6}$ Estos y otros trabajos pusieron de manifiesto que la raza, la etnicidad y el género son socialmente construidas, constituyendo no sólo identidades individuales sino también principios de organización del sistema social. ${ }^{7}$

En este marco, las feministas negras cuestionaron a aquellas blancas el modo de teorización de la 'mujer' y sus subordinaciones. Como explica Lugones, el feminismo blanco:

\begin{abstract}
centraba su lucha y sus maneras de conocimiento y teorización contra una caracterización de la mujer como frágil, débil de cuerpo y mente, recluida en lo privado y sexualmente pasiva. Pero no llegó a hacerse consciente de que esas características construían sólo a las mujeres blancas burguesas. En realidad, al comenzar desde esa caracterización, las feministas blancas burguesas teorizaban a la femeneidad blanca como si todas las mujeres fueran blancas. Comprendían a las mujeres como dotadas de un cuerpo blanco pero no llevaban esa cualificación racial a una articulación o a una conciencia clara. Es decir, no se comprendían a sí mismas en términos interseccionales, en una intersección de raza, de género y de otras señales fuertes de sujeción o de dominación. Y porque no percibían esas profundas diferencias, no veían la necesidad de crear coaliciones. Suponían una hermandad, un vínculo que venía dado con la sujeción del género. ${ }^{8}$
\end{abstract}

Ahora bien, aunque Crenshaw introduce el término interseccionalidad a fines de la década del ochenta, esto no implica que fuera la primera en abordar la exclusión de las mujeres negras y la forma en que habían sido distorsionadas dentro del discurso feminista dominante. Ya en 1977, un grupo de feministas lesbianas negras en Estados 
9 Kathy DAVIS, 2008; Stephanie SHIELDS, 2008. De reciente edición en español se encuentra una interesante compilación sobre los feminismos negros (Mercedes JABARDO, 2012).

${ }^{10}$ Adriana PISCITELLI, 2008, p. 267.

1 Raquel PLATERO, 2012, p. 46.

${ }^{12}$ ANTHIAS, 2006, p. 14-15.

${ }^{13}$ Verena STOLCKE, 2004, p. 96.

${ }^{14}$ DAVIS, 2008, p. 70.

15 ANTHIAS y Nira YUVAL DAVIS, 1983.

${ }^{16}$ BROWNE y MISRA, 2003.
Unidos reclamaron que el género, la raza, la clase y la sexualidad debían ser parte integral de cualquier análisis feminista sobre poder y dominación. ${ }^{9}$ Así pues, el concepto de interseccionalidad que se fue construyendo con diferentes términos a lo largo del tiempo (simultaneidad, matriz de dominación, ejes de desigualdad, agenciamientos, posiciones de ubicación, vectores de poder, categorías de articulación) y fue involucrando la coexistencia de distintos abordajes, ${ }^{10}$ permitió también visibilizar la imbricación de los movimientos sociales con la academia. ${ }^{11}$

La apuesta interseccional, al disputar la noción de una posición política atada a una forma singular de identidad (por ejemplo, género, etnicidad, raza, clase social, etc.), ${ }^{12}$ se asienta en la necesidad de recuperar las experiencias de los grupos subordinados y las relaciones de poder que enfrentan en diversos contextos socio-históricos. Pero, además, la interseccionalidad viene a complejizar la concepción de género al concebirla una dimensión entre otras dentro del complejo tejido de las relaciones sociales y políticas. ${ }^{13}$ En tal sentido, retoma cuestiones centrales del pensamiento feminista contemporáneo, como es el conocimiento de los efectos que diferentes formas de clasificación social tienen sobre la identidad y las experiencias de las mujeres, así como en su lucha por transformar las relaciones de poder. ${ }^{14}$ Para ello, asume la difícil tarea de los desenmascaramientos: de formas de opresión, de falsos universalismos, de múltiples clasificaciones sociales y su incidencia en la vida de las personas.

Inicialmente, la teoría feminista adoptó el enfoque de la 'triple discriminación' en relación con la clase, el origen étnico-racial y el género, explorando cómo frente a cada nueva forma de clasificación social la persona se volvía más vulnerable y subordinada. Gradualmente, el eje pasó a ser el modo en que el género, el origen étnico-racial y la clase, entre otras posibles clasificaciones sociales, interactúan y se imbrican en las realidades sociales y materiales de la vida de las mujeres, configurando determinadas relaciones de poder. ${ }^{15}$ En este marco, la perspectiva interseccional sugiere que no existe una percepción de género que sea racial y étnicamente ciega, a la vez que no existe una percepción étnica y racial que sea genéricamente ciega. Al contrario, las percepciones están basadas en una constitución de atributos sociales de los individuos en el marco de la interacción -etnicidad, género, clase- antes que en una única dimensión. ${ }^{16}$

Desde las últimas décadas, las elaboraciones teóricas sobre interseccionalidad alcanzaron un lugar relevante en el campo de los estudios feministas. En uno de sus trabajos, Davis postula que su 'éxito' se debe a cuatro aspectos

694 Estudos Feministas, Florianópolis, 23(3): 691-712, setembro-dezembro/2015 
17 DAVIS, 2008

18 Mara VIVERO VIGOYA, 2009; Chandra MOHANTY, 2008.

19 DAVIS, 2008, p. 73.

${ }^{20}$ Ann BRAH y Ann PHOENIX, 2004, p. 82.

21 DAVIS, 2008; Jennifer NASH, 2008; PHOENIX Y Pamela PATTYNAMA, 2006. fundamentales. ${ }^{17}$ El primero de ellos radica en el hecho de que la interseccionalidad aborda una de las preocupaciones centrales para ese campo de estudios como son las diferencias entre las mujeres, desafiando el modelo hegemónico de mujer universal. ${ }^{18}$ Si bien el punto de partida ha sido la disputa de ese modelo hegemónico, esto no significa que interseccionalidad sea sinónimo de mujeres, por el contrario, las experiencias de los varones también se hallan condicionadas por las intersecciones de clasificaciones sociales y, por ende, resultan un objeto de indagación central (aunque menos explorado aún) de esta perspectiva.

El segundo aspecto reside en que provee un 'toque' novedoso a un viejo problema. A pesar que la interseccionalidad aborda un viejo problema dentro de las investigaciones feministas, como es la cuestión del poder y las lógicas de dominación, lo hace con una nueva plataforma fundada en una articulación novedosa entre la teoría feminista crítica sobre los efectos del sexismo, del racismo y de la clase social; y la metodología crítica inspirada en el teoría feminista posmoderna. ${ }^{19}$ Dicha perspectiva recupera las preocupaciones en torno a las consecuencias sociales y materiales de las categorías género, etnicidad, raza y clase a la vez que incorpora metodologías compatibles con el proyecto postestructuralista de la necesidad de deconstrucción de ciertas categorías, de desenmascarar los falsos universalismos y de identificar las dinámicas de poder imperantes. ${ }^{20} \mathrm{El}$ tercer aspecto consiste en que la interseccionalidad ha interpelado a una amplia audiencia académica, proponiendo herramientas para describir ideas que se convirtieron en centrales dentro del pensamiento feminista y del campo de los estudios de mujeres como son los mecanismos de producción y reproducción de múltiples desigualdades y formas de dominación. Por último, el cuarto aspecto se asienta en la propia ambigüedad que la interseccionalidad presenta, en tanto al no resultar una enfoque teórico cerrado requiere de más síntesis y elaboraciones, proponiendo nuevos desafíos a los/as investigadores/as sensibles a las problemáticas vinculadas con el poder y las desigualdades. ${ }^{21}$ Bajo este escenario, la 'vitalidad' de la interseccionalidad se ha nutrido, fundamentalmente, de tres aportes principales. En primer lugar, y vinculado estrechamente al sentido de su surgimiento, reflexiona acerca de las experiencias de los grupos subordinados y las relaciones de poder que estructuran las sociedades, no subsumiéndolas a una sola forma de clasificación social, a la vez que contempla el modo en que los sujetos cuestionan y resisten las lógicas de opresión y subordinación. En segundo lugar, considera que el género, la etnicidad, la raza y la clase social, entre otras clasificaciones sociales, operan en múltiples 
${ }^{22}$ Mieke VERLOO, 2006, p. 213.

${ }^{23}$ PHOENIX, 2006, 187; SHIELDS, 2008 , p. 301.

${ }^{24}$ Karina BIDASECA, 2010; Ochy CURIEL, 2007; VIVERO VIGOYA, 2009.

\begin{abstract}
${ }^{25}$ Si bien no podemos detallar aquí la vasta producción en torno a estos temas elaborada en Brasil, es preciso mencionar las investigaciones pioneras de Lelia GONZÁLEZ, 1984, 1988; Heleieth SAFFIOTI, 1992; Luiza BARRIOS, 1995; Mary GARCÍA CASTRO, 2001. Asimismo, es necesario destacar la visibilidad de la interseccionalidad en espacios de divulgación como el Seminario Internacional Fazendo Gênero (desde el año 1994) y la Revista Estudos Feministas (desde el año 1992), entre otros.

26 Algunos de los principales referentes de esta discusión son: Ramón GROSFOGUEL, 2007; LUGONES, 2008; Walter MIGNOLO, 2007; Aníbal QUIJANO, 2000; Eduardo RESTREPO y Axel ROJAS, 2010; Catherine WALSH, 2008. ${ }^{27}$ GROSFOGUEL, 2007, p. 100.
\end{abstract}

niveles de la vida social, configurando una base material e ideológica. En este sentido, la interseccionalidad es entendida a nivel de grupos y sujetos y también a nivel estructural, dando cuenta de las bases materiales de la desigualdad. ${ }^{22}$ En tercer lugar, establece que la producción de conocimiento debe considerar las posiciones y clasificaciones sociales de manera relacional, complejizando los enfoques que intentan reducir las experiencias de los sujetos bajo el lente de una sola categoría. ${ }^{23}$

En América Latina, específicamente, el camino trazado por la interseccionalidad tuvo sus inicios en la década del ochenta aunque se fue consolidando como perspectiva teórico-metodológica recién en el último decenio. En el transcurso de este período, surgieron una serie de trabajos desde el feminismo poscolonial y transnacional que buscaron poner en discusión las implicancias históricas y políticas de lógicas de dominación de género, sexuales, raciales y clasistas. ${ }^{24} \mathrm{El}$ caso de Brasil muestra algunas diferencias en comparación al resto de la región, pues los estudios sobre interseccionalidad presentan un mayor desarrollo vinculado principalmente al lugar que han ocupado los estudios de género, raza y colonialidad en el ámbito social, político y académico nacional. ${ }^{25}$ Las investigaciones que fueron surgiendo en torno a aquellas preocupaciones (lógicas de dominación de género, sexuales, raciales y clasistas) pretendieron disputar las relaciones de poder históricamente situadas en el contexto de las discusiones sobre la pervivencia de la matriz colonial del poder en América Latina. ${ }^{26}$ El punto central de estos marcos teóricos ha sido la localización geopolítica y corpo-política del sujeto que habla/enuncia en las coordenadas del poder global. ${ }^{27}$ En esta localización, el género, la etnicidad, la raza, la sexualidad y la clase social resultan componentes claves de la epistemología y la producción de conocimiento.

\section{Los 'problemas' de la interseccionalidad}

La centralidad que ha adquirido la perspectiva interseccional ha estado acompañada por una serie de dudas y cuestionamientos acerca del modo de operativizar en términos metodológicos sus argumentaciones teóricas. A manera de síntesis, podríamos resumir en cuatro los 'problemas' que presenta la interseccionalidad: el abordaje metodológico; la (in)definición respecto al sujeto de la interseccionalidad y el hecho de tomar las categorías (género, etnicidad, raza, clase social, sexualidad, etc.) como dadas; el potencialmente ilimitado número de categorías interseccionales; y la confusión entre identidades interseccionales y estructuras interseccionales. 
${ }^{28}$ MCCALL, 2005; NASH, 2008.

${ }^{29} \mathrm{NASH}, 2008$, p. 6.

${ }^{30}$ ANTHIAS, 2006, p. 65.

${ }^{31}$ Ana Inés MALLIMACl, 2013, p. 92.
En relación con el primero de los 'problemas', diversos estudios enfocados en pensar y reflexionar sobre la interseccionalidad, sus aportes y limitaciones, coinciden en señalar que ha habido una escasa discusión respecto a cómo estudiarla, haciendo especial referencia a la cuestión metodológica. ${ }^{28}$ En particular, las dificultades surgen cuando se intenta analizar empíricamente la manera en que opera la simultaneidad de las diferentes clasificaciones sociales (clase, género, etnicidad, origen nacional, raza, entre otras) en determinados grupos sociales. Por otra parte, y vinculado siempre a la cuestión metodológica, una de las críticas a la interseccionalidad se asienta en la distancia entre las estrategias metodológicas para problematizar la interseccionalidad y las prácticas de investigación sustentadas en esta perspectiva. Específicamente, una de las tensiones reside en el hecho de que mientras la perspectiva interseccional ha trabajado en pos de discutir aquellos enfoques que conciben a las identidades en términos aditivos (por ejemplo género + raza + etnicidad + sexualidad + clase = una identidad compleja) y de reflexionar sobre los procesos sociales de categorización a través de las estrategias desplegadas por los sujetos en contextos de exclusión y opresión; los proyectos basados en la perspectiva interseccional a menudo replican la aproximación que critican. ${ }^{29}$ Una persona no enfrenta lógicas de exclusión a partir de la suma de ejes de desigualdad sino que su propia posición se constituye por la intersección de esos ejes. Es por ello que se subraya la necesidad de evitar el modelo aditivo a la hora de poner en juego los elementos teóricos de la interseccionalidad, reconociendo que el género, la etnicidad, la raza y la clase, entre otras, son clasificaciones sociales producidas interseccionalmente. Es en este sentido que se sugiere pensar al género como etnizado, siempre racializado, siempre influido por la clase y así sucesivamente. ${ }^{30}$

El segundo de los 'problemas' que plantea la interseccionalidad radica en el riesgo de considerar como dadas las categorías mediante las cuales se intenta identificar y comprender cómo funcionan las lógicas de opresión, desigualdad y resistencia. Para evitar esto, es necesario retomar las categorías de análisis a partir del grupo social objeto de estudio y del contexto histórico específico. Es decir, las clasificaciones sociales que condicionan las experiencias de vida de las personas remiten a un marco espacial y temporal concreto pues "las categorías de clasificación y nominación configuradas nacionalmente le confieren diversos significados históricos a lo 'racial', lo 'nacional' y lo 'étnico'. ${ }^{31}$ De este modo, no existe una única forma de interseccionalidad sino varias dependiendo del 
32 En los trabajos producidos desde la interseccionalidad en los países del 'norte', en especia anglosajones, la noción de raza prevalece a la de etnicidad. Sin embargo, aquellos trabajos realizados en gran parte de América Latina, con excepción de Brasil, y España, la etnicidad cobra protagonismo frente a la forma de clasificación raza. El uso de ambos conceptos no está exento de tensiones y contradicciones. La noción de etnicidad, utilizada para designar una comunidad discreta caracterizada por rasgos comunes, es relativamente más nueva que la noción de raza, difundiéndose ampliamente durante la segunda posguerra. La raza, al igual la etnicidad, son construcciones simbólicas que se utilizan en ciertas circunstancias socio-políticas como criterio de definición y delimitación de grupos humanos (STOLCKE, 2000 , 41)

${ }^{33} \mathrm{NASH}, 2008$, p. 9-10.

${ }^{34}$ Cynthia ANDERSON, 1996, p. 734.

35 YUVAL-DAVIS, 2006, p. 201. grupo social a estudiar y del universo socio-histórico particular. Relacionado con este punto emerge el interrogante respecto a quién es el 'sujeto interseccional', si es que existe tal sujeto. Como sugieren distintos estudios, las investigadoras feministas no se han puesto de acuerdo al respecto. Por un lado, un grupo más reducido de feministas considera que la interseccionalidad hace referencia a todas las posiciones del sujeto, las cuales son fundamentalmente constituidas por el género, la raza, la sexualidad y la clase social. ${ }^{32}$ Por otro lado, la mayor parte de los estudios sobre interseccionalidad se ha focalizado en las posiciones particulares de aquellos sujetos que enfrentan formas de opresión y exclusión. ${ }^{33} \mathrm{En}$ tal sentido, el sujeto objeto de análisis privilegiado ha sido el oprimido, el excluido, aquel sobre el cual recaen las lógicas de dominación y desigualdad, el 'otro'. Esto, sin embargo, no significa desconocer las potencialidades de la interseccionalidad para pensar a distintos grupos sociales, se ubiquen en posiciones de subordinación o dominación, teniendo en cuenta además que un sujeto puede ocupar a la vez distintas posiciones, algunas de ellas de subordinación y otras de dominación. Lo importante, según subraya Anderson, es que las categorías interseccionales (género, raza, clase son las que retoma la autora) deben conceptualizarse como procesos dinámicos y fluidos que se encuentran insertos en contextos sociales, espaciales y temporales específicos. ${ }^{34}$ Esta premisa, que supone que las clasificaciones sociales operan en un escenario socio-histórico determinado, permitirá escapar de las visiones que las conciben como dadas, fijas e inmutables.

El tercer 'problema' de la interseccionalidad es aquel referido al potencialmente ilimitado número de categorías de análisis. Este 'problema' se asienta en encontrar respuestas a la cuestión de cuáles y cuántas son las clasificaciones sociales que se pueden recuperar para reflexionar teórica y empíricamente acerca de las desigualdades múltiples y las identidades complejas. Es posible identificar un consenso bastante generalizado en torno al género, la raza/etnicidad y clase social como las clasificaciones sociales con mayor peso en la estructura de relaciones sociales contemporáneas. ${ }^{35}$ A estas clasificaciones, algunas/os estudiosas/os incluyen la edad, la sexualidad, el origen nacional, la discapacidad, entre otras. Ese 'entre otras' es, justamente, una de las mayores tensiones de la interseccionalidad pues remite al interrogante - aún no resuelto - sobre el número - máximo y mínimo - de categorías interseccionales con el cual trabajar, en términos teóricos y metodológicos. En líneas generales, las clasificaciones sociales 'principales' (género, raza, etnicidad, clase) poseen ciertos parámetros en común, en especial, el hecho de que tienden a ser naturalizadas y percibidas como resultado de un destino biológico asociado con determi- 
${ }^{36}$ YUVAL-DAVIS, 2006, p. 198.

37 YUVAL-DAVIS, 2006.

${ }^{38}$ ANDERSON, 1996, p. 736.

${ }^{39}$ Joan ACKER, 1992, p. 567. Esta autora recupera la noción de 'instituciones generizadas' para hacer referencia al hecho de que el género se encuentra presente en los procesos, prácticas, imágenes, ideología y relaciones de poder en distintas esferas de la vida social.

${ }^{40}$ ANTHIAS, 2012, p. 107 nadas características de los grupos sociales. ${ }^{36}$ Esto se vincula con el debate existente en torno al modo en que se 'produce' la intersección y a la cuestión, todavía no cerrada, respecto a si siempre el género, la etnicidad, la raza y la clase social se intersectan. Respecto a esto último, es preciso subrayar que las clasificaciones sociales presentan diferentes lógicas de organización, aun cuando se las naturalice y se las conciba a partir del mismo lente. Lo importante, según establece Yuval-Davis, es que para ciertos grupos sociales existen situaciones históricas específicas que condicionan que determinadas clasificaciones sociales sean más importantes que otras en la construcción de posiciones y relaciones sociales concretas. ${ }^{37}$

El cuarto de los 'problemas' de la interseccionalidad se relaciona con la confusión entre identidades interseccionales y estructuras interseccionales. Este 'problema' se funda en la tensión entre las identidades sociales de los sujetos y la estructura social que emerge de la interseccionalidad. Anderson, por ejemplo, pretende resolver esta tensión planteando que cualquier análisis de la desigualdad social debe considerar a los individuos y a las estructuras, en tanto el género, la raza y la clase son relaciones de poder que crean, nutren y modifican los sistemas de organización social estando, por ende, insertos en las estructuras. Bajo esta premisa, resalta que es preciso examinar el movimiento entre género, clase, raza como principios organizadores de la identidad social y de la estructura social. ${ }^{38}$ Esta última no está desprovista de clasificaciones sociales, por el contrario, las leyes, la política, la religión, la academia, el estado y el mercado de trabajo se encuentran constituidos por el género, la etnicidad, la clase social, la raza. ${ }^{39}$ En el intento de superar las visiones dicotómicas entre identidades y estructuras interseccionales, Anthias propone analizar la interseccionalidad en términos de estructuras (en referencia a las marcas económicas y políticas) y en términos de procesos (las relaciones sociales en toda su complejidad, incluyendo discursos y representaciones). ${ }^{40}$

Ahora bien, los 'problemas' que presenta la interseccionalidad no invalidan sus aportes y potencialidades para pensar las desigualdades sociales a nivel de grupos $y$ estructuras, y comprender la diversidad de trayectorias y experiencias de los/as sujetos. Por el contrario, invitan a reflexionar sobre posibles trazados teóricos y metodológicos que permitan consolidar la interseccionalidad como una perspectiva clave para el estudio de los sujetos sociales y sus experiencias de desigualdad y resistencia, y también de las estructuras y sus lógicas de organización. Estos 'problemas' pueden reconfigurarse como caminos abiertos, preguntas aún sin respuestas que no clausuran lo que la interseccionalidad 


\begin{abstract}
${ }^{42}$ Véase, entre otros, los trabajos de: ANTHIAS, 2006 y 2012; BROWSE Y MISRA, 2003; HansJoachim BÜRKNER, 2012; Sandra EZQUERRA SAMPER, 2008; Pierrette HONDAGNEU-SOTELO, 2007; María José MAGLIANO, 2013; MALLIMACI, 2011; Sonia PARELLA, 2003; Patricia PESSAR y Sarah MAHLER, 2003; Adriana PISCITELLI, 2008 y 2009; María Gabriela POMBO, 2011.

${ }^{43}$ HONDAGNEU-SOTELO, 2007, $\mathrm{p}$. 426.
\end{abstract}

${ }^{44}$ ANTHIAS, 2006, p. 66.

${ }^{45}$ ANTHIAS, 2012, p. 102 vino a decir al campo de las ciencias sociales. Son esos mismos 'problemas', que se traducen en una serie de desafíos para los y las investigadores/as, los que le pueden augurar una larga vida a la interseccionalidad.

\section{Hacia un análisis interseccional de trayectorias laborales de mujeres migrantes en Córdoba}

Los procesos migratorios, al estar conformados por diversos ejes de desigualdades, resultan un campo relevante para el análisis teórico y empírico de la interseccionalidad. En las migraciones internacionales, las clasificaciones de género, clase, origen nacional, raza, etnicidad, edad, condición migratoria y religión pueden incidir directamente en la vida cotidiana de mujeres y varones e influir de manera determinante en su acceso a derechos y oportunidades, así como en las situaciones de privilegio o de exclusión que de ellas se derivan. ${ }^{41}$

Los estudios sobre interseccionalidad en el campo de las migraciones internacionales reconocen al género como una dimensión central para analizar experiencias y trayectorias migratorias a la vez que consideran que ésta no es una dimensión aislada sino uno de los diversos procesos, en intersección con la etnicidad y la clase social, entre otras clasificaciones, que crean y perpetúan desigualdades sociales. ${ }^{42}$ Como afirma Hondagneu-Sotelo: "el género no existe de forma aislada sino que es siempre parte de un esquema en que la raza, la nacionalidad, la integración ocupacional y las posiciones de clase socioeconómica se relacionan de modo particular, y el análisis de todo ello refleja los matices de dicha interseccionalidad". ${ }^{43}$

Dentro de los estudios migratorios, Floya Anthias ha reflexionado en profundidad sobre la complejidad y la diversidad de experiencias de los y las migrantes. Es sus textos, enfatiza la centralidad de retomar la dimensión de género pensando también en su sentido etnizado, racializado y de clase; al mismo tiempo que subraya que no es posible comprender los sentidos de la etnicidad y de la migración sin tener en cuenta el género y la clase. ${ }^{44}$

Asimismo, señala la necesidad de que los estudios se enmarquen dentro de análisis procesuales, contextualizados y dinámicos que reconozcan la ilimitada interconexión de las diferentes identidades y estructuras jerárquicas en relación al género, la etnicidad, la clase y otras clasificaciones sociales a nivel local, nacional, transnacional y global. ${ }^{45}$ Esto supone tener en cuenta el alcance y las significaciones no sólo de las fronteras jurídico-administrativas que delimitan un Estado nacional, sino también las múltiples fronteras interiores que 
47 Gioconda HERRERA, 2012 y 2013.

${ }^{48}$ Ina KERNER, 2009, p. 193
${ }^{46}$ PISCITELLI, 2007, 2008, 2009.

configuran clasificaciones sociales, tanto en el país de destino como en el de origen de los/as migrantes.

En la esfera latinoamericana sobre migraciones, en particular, la perspectiva interseccional ha alcanzado mayor visibilidad en los últimos años, aunque aún no ha superado la etapa de enunciación teórica. Algunas excepciones son los trabajos de Adriana Piscitelli, centrados fundamentalmente en las experiencias de migrantes brasileras, en especial vinculadas al mercado del sexo y en los cuales recupera una perspectiva feminista transnacional atenta a las diversas categorías de articulación e interseccionalidades que atraviesan sus experiencias y trayectorias; ${ }^{46}$ y de Gioconda Herrera, focalizados en diferentes aspectos de la migración ecuatoriana transnacional y las cadenas globales de cuidado. ${ }^{47}$

En el campo de investigación sobre migración y trabajo, la interseccionalidad puede resultar potencialmente útil para examinar los sentidos e implicancias del mercado laboral en la vida de los/as migrantes, en tanto la articulación de clasificaciones sociales (sean de género, etnicidad, clase, sexual, etc.) puede producir subordinación respecto al trabajo a la vez que justificar y naturalizar la inserción de los/as migrantes en actividades específicas, en general inestables, mal pagas e informales. ${ }^{48}$ En relación con el último objetivo del artículo, proponemos como desafío teórico-metodológico analizar desde la interseccionalidad trayectorias laborales de mujeres peruanas migrantes que se dedicaron en algún momento de su experiencia migratoria al trabajo doméstico remunerado, partiendo de la premisa que las indagaciones empíricas desde esta perspectiva suponen una selección de determinadas clasificaciones y categorías de articulación, fundada en el reconocimiento de un marco social, espacial e histórico específico. En este caso en particular, el género, la clase social, el origen nacional, la edad y la condición migratoria emergen como clasificaciones sociales principales, produciendo interseccionalmente diferentes trayectorias $y$ experiencias al interior del trabajo doméstico remunerado. ${ }^{49}$

La migración peruana hacia Argentina en general y

do comprende las tareas de limpieza, cocina y cuidado general de la vivienda, y la asistencia y cuidado no terapéutico de personas (desde niños hasta ancianos). Las mujeres migrantes lo realizan fundamentalmente bajo dos modalidades principales: cama adentro y externo fijo o por horas.

${ }^{50}$ Marcela CERRUTTI, 2005; María del Carmen FALCÓN AYBAR y Eduardo BOLOGNA, 2013 Carolina ROSAS, 2010.
Córdoba en particular está relacionada con la búsqueda de mejores oportunidades socio-económicas. Se trata de un flujo que se magnificó en los años noventa, joven, con una alta presencia femenina concentrada en zonas urbanas, sobre todo Buenos Aires, Córdoba y Mendoza. ${ }^{50}$ Para un amplio conjunto de migrantes peruanas, el trabajo doméstico remunerado se ha constituido en la actividad laboral más importante en torno al cual se orientaron y organizaron dinámicas migratorias individuales y familiares. En Argentina, la concentración de mujeres peruanas en este 
sector se ha asentado en el presupuesto extendido de su 'sobrecalificación' en comparación con las 'otras' mujeres que también se desempeñan en esta tarea, como son las migrantes internas y de otros países de la región sudamericana, en especial Bolivia y Paraguay.

En el trabajo de campo realizado en la ciudad de Córdoba hemos podido reconstruir distintas trayectorias y experiencias de trabajadoras domésticas peruanas. Los puntos de encuentro en las trayectorias de estas mujeres resultan la condición de género (todas ellas son mujeres); el origen nacional (todas ellas nacieron en Perú); el origen urbano de la migración (la inmensa mayoría proviene de centros urbanos del país de origen); la juventud (todas ellas arribaron para trabajar como empleadas domésticas entre los 20 y 25 años de edad); las posibilidades de inserción en el trabajo doméstico remunerado se activan a partir de la migración (la gran mayoría de estas mujeres no se dedicaba a esta ocupación en su país de origen); la maternidad y los hijos actúan sobre las posibilidades y modalidades de inserción laboral al mismo tiempo que pueden incidir en la movilidad hacia otro sector laboral; y han sido pioneras de la migración (son las mujeres quienes encabezaron el proyecto migratorio). Sin embargo, la reconstrucción de estas trayectorias desde una perspectiva que contemple las diversas clasificaciones sociales interseccionalmente nos permite vislumbrar diferencias en las experiencias laborales de estas mujeres a partir de la pertenencia de clase (vinculada especialmente a la calificación laboral y al nivel de escolaridad alcanzado), la condición migratoria y las dinámicas familiares, las cuales redundan en distintos proyectos y realidades migratorias. Las trayectorias de Daniela, Gisella y Raquel ilustran estas diferencias.

Daniela llega a Córdoba en el año 1996, en el período en que comienza a profundizarse el proceso migratorio peruano hacia Argentina, con un grupo de amigos, dejando en Lima a su hija de siete años (con quien nunca vuelve a convivir pues siempre permaneció en Perú). Si bien Daniela 'no llega' con un empleo ya estipulado, a los pocos días comienza a trabajar como empleada doméstica cama adentro. Gisella, por su parte, arriba a la ciudad en el año 2002 para trabajar como empleada doméstica cama adentro, dejando en Lima a su hija, Mariana, que tenía en aquel momento 7 años. Raquel llega en el año 2010, con una amiga, directamente desde Lima. En su Perú natal, Daniela era asistente social y había cursado estudios de educación primaria en la Universidad de la Cantuta. Al momento de migrar, trabajaba en el área social de la Municipalidad en Lima. Gisella había completado la escuela media y trabajaba en Lima vendiendo y fabricando 
51 Ania TIZZIANI, 2011.

\footnotetext{
52 Daniela, Córdoba, 2014.
}

\begin{abstract}
${ }^{53}$ Daniela, Córdoba, 2014.
${ }^{54}$ En el año 2004 , con la sanción de la Ley $\mathrm{N}^{\circ} 25.871$, se modifica la legislación migratoria vigente desde la última dictadura militar (1976-1983). A partir de ese momento, el acceso a la regularidad migratoria no necesariamente se encuentra atado a la formalidad laboral. Sin embargo, los niveles de exclusión y explotación a partir de la informalidad y precariedad laboral pueden atentar contra la posibilidad de regularizar la situación migratoria.

${ }^{55}$ Daniela se casa con un migrante boliviano y tiene dos hijos nacidos en Córdoba.
\end{abstract}

exhibidores de indumentaria en una jornada laboral que se extendía por 12 horas diarias. Finalmente, Raquel no había finalizado sus estudios secundarios y se dedicaba a la venta informal en la capital peruana. A pesar de que las tres poseen diferentes experiencias y trayectorias pre migratorias, la primera inserción laboral en Córdoba - e incluso motor de la migración - fue el trabajo doméstico remunerado. De algún modo, esta actividad aparece como una elección que cobra sentido cuando se lo integra dentro del universo de inserciones laborales posibles en el lugar de destino. ${ }^{51}$ Asimismo, al migrar solas - Daniela y Gisella como pioneras de la migración (dejando a su familia en el lugar de origen) -, la modalidad cama adentro se convirtió en los inicios en la mejor opción en tanto les permitía ahorrar la máxima cantidad de dinero posible.

De acuerdo con el testimonio de Daniela:

Yo me vine sola con un grupo de amigos, la señora que nos trajo era amiga de mi hermano. En esa época trabajaba en el ministerio en la Municipalidad de Lima como Asistente Social, entonces me ofrecieron una mejor vida, una posibilidad de crecer, me pintaron pajaritos y cuando yo llegue acá nada de pajaritos. Los pajaritos se murieron todos porque me llevaron a una pensión y a los quince días la señora que nos trajo nos abandonó, ahí tuvimos que dejar la pensión. Fue una historia muy dura pero, si me volvía, fracasaba al no irme con algo. Bueno comencé a conocer a mucha gente, soy muy habladora, pude encontrar otro lugar y otro espacio donde poder estar porque yo no tenía un techo, me vine con mi bolsita, mi ropa y eso era todo. ${ }^{52}$

Al poco tiempo de su llegada a Córdoba, Daniela comienza a trabajar como empleada doméstica para asegurar su subsistencia y la de su hija que había quedado en Lima: "yo trabajaba muy bien y ganaba en esa época, giraba dinero y, a la vez, yo mantenía el alquiler, la comida del mes, trabajaba cama adentro, por supuesto, entonces de esa forma ganaba pero también era todo un sacrificio". ${ }^{53}$

El hecho de trabajar de manera formal ('en blanco') le posibilitó a Daniela acceder a la regularidad migratoria en los años noventa, cuando la legislación exigía más requisitos a los/as migrantes para la obtención de la documentación. ${ }^{54}$ Desde sus inicios como empleada doméstica, Daniela trabajó varios años para los mismos empleadores (9 años para uno y 14 años para otro). Los primeros años lo hace cama adentro para luego, a partir de la maternidad, pasar a trabajar de manera externa fija por horas. ${ }^{55}$ El problema para Daniela comienza cuando uno de sus empleadores la quiere despedir sin la correspondiente 
indemnización. En el año 2012 se acerca al Sindicato del Personal de Casas de Familia (SIN.PE.CAF) para asesorarse y decidir qué caminos seguir. A raíz de esto, toma la decisión de llevar a juicio a su empleador (con el que había trabajado por 14 años) a la vez que comienza a participar visible y activamente en esa institución, informando y asesorando a sus 'compatriotas' respecto a sus derechos y obligaciones como trabajadoras domésticas.

Por su parte, cuando Gisella llega directamente para trabajar como empleada doméstica cama adentro en uno de los barrios más acomodados de la ciudad, tenía a sus dos hermanas viviendo y trabajando bajo esa modalidad allí. De hecho, son ellas quienes le consiguen el trabajo: "llegué un sábado por la tarde y el domingo ya estaba trabajando, ya me había metido cama adentro". ${ }^{56}$ Este no es un fenómeno aislado, al contrario, la gran mayoría de las trayectorias laborales reconstruidas pone de relieve que la migración se asienta en redes sociales de contacto e información entre origen y destino que facilitan la llegada y la inserción laboral. A diferencia de Daniela, Gisella permanece todo un año trabajando para la misma empleadora de manera informal e indocumentada, enviando casi todo el dinero que obtenía a Lima para la manutención de su hija:

No aguanté más sin mi hija y al año me regresé. La señora para la que trabajaba me compró el pasaje para irme, de ida y vuelta, para que viera a mi hija, pero yo ya no volví. Creo que me había agarrado cariño, al principio no, pero después me conoció. Ya me quedé unos dos años en Lima, y luego nos vinimos todos ya, mi esposo (que no es el padre de Mariana) y mi hija, pero ya no trabajé más en casas, me quedé cuidando a mi hija y Roberto encontró trabajo en la construcción. ${ }^{57}$

En el caso de Raquel, al momento del arribo a Córdoba comienza a trabajar como empleada doméstica cama adentro en una zona residencial de la ciudad por un salario muy bajo: "como no tengo documentos me pagaban poco y en negro", nos comentó. ${ }^{58}$ Desde su llegada, Raquel siempre se mantuvo en condición de irregularidad migratoria, siendo este un factor condicionante de sus trayectorias laborales en Córdoba. A comienzos del año 2012 queda embarazada y es despedida de su trabajo. Ahí comienza un largo peregrinaje en busca de un empleo que le permita subsistir. Así, trabaja en casas por horas, siempre de manera informal e indocumentada, hasta que tiene a su hijo. Como el padre del niño (también de origen peruano) no aporta a su manutención, Raquel se vale de la solida- 
${ }^{59}$ En Córdoba, la costura concentra a un importante conjunto de migrantes peruanos, varones $y$ mujeres.

${ }^{60}$ Administración Federal de Ingresos Públicos.

${ }^{61}$ Raquel, Córdoba, 2013.

${ }^{62}$ En relación con esto, es preciso destacar que la histórica informalidad del trabajo doméstico remunerado incluye tanto a mujeres migrantes como nativas, aunque en el caso de las primeras esta situación ha sido tradicionalmente más generalizada. En abril del año 2013 se aprobó en Argentina una nueva ley (Ley $\mathrm{N}^{\circ} 26.844$ ) que busca regular a esta actividad, siendo uno de sus principales propósitos la 'formalización' laboral de las trabajadoras domésticas. Debido a lo reciente de la ley, aún no hay datos fehacientes respecto a su impacto en esa dirección, aunque se presume un cambio positivo en cuanto al porcentaje de trabajadoras domésticas que pudieron regularizar su situación laboral.

${ }^{63}$ De acuerdo al relato de Daniela el pasaje de empleada doméstica al trabajo en seguridad es considerado una mejora sustancial y un 'ascenso' en relación con sus posibilidades laborales, vinculado ello a otro pasaje: el de una actividad concebida tradicionalmente como 'femenina' (trabajo doméstico remunerado) a otra constituida como 'masculina' (seguridad).

${ }^{64}$ Gisella tiene en la actualidad tres hijos: Mariana, quien nació en Perú, Sara y Ramiro, quienes nacieron en Córdoba fruto de su relación con Roberto. ridad de sus 'compatriotas' (desde el momento en que queda embarazada vive en diferentes casas de familias peruanas). Una vez que se recupera del parto comienza a buscar un nuevo trabajo. En un primer momento, lo encuentra en un lugar de comidas, en el mismo barrio donde residía. Sin embargo, la relación entre el tiempo de trabajo (casi 12 horas por día) y el estipendio recibido (150 pesos semanales) la impulsa a buscar otra ocupación. Entre las mujeres migrantes peruanas - y también los varones - hay pocas opciones de inserción laboral, siendo una de ellas la costura. ${ }^{59}$ Así, consigue un trabajo en un taller en donde además alquila una pieza - compartida con otros 'compatriotas' - para ella y su hijo, siempre de manera informal e indocumentada. En relación con su propia experiencia laboral en Córdoba, Raquel concibe que "el trabajo doméstico es más estable, eso es lo que yo vine a hacer y quiero hacer, trabajar en casas de familia. El trabajo en costura es más peligroso porque vienen seguido los de la AFIP60 a controlar y, como estoy indocumentada, vivo asustada". ${ }^{61}$

Es importante subrayar que la posibilidad de la formalidad laboral y de lograr mejores condiciones de trabajo no es una cuestión de voluntad de las trabajadoras migrantes sino que más bien se vincula a la inestabilidad y explotación que caracterizan a las inserciones principales a las que acceden muchos/as migrantes peruanos/as - y no sólo ellos/as -; sin embargo, ciertos saberes adquiridos tanto en origen como en destino resultan centrales a la hora de poder enfrentar y resistir algunas de esas inestabilidades y explotaciones. ${ }^{62}$ Los casos analizados dan cuenta de ello. En la actualidad, Daniela trabaja como seguridad para un sindicato, de manera formal, mientras aguarda por la finalización del juicio laboral con su antiguo empleador (como trabajadora doméstica); ${ }^{63}$ Gisella posee un pequeño kiosco que atiende en su casa conjuntamente con su hija mayor mientras su marido se dedica a la construcción, ${ }^{64}$ Raquel continúa transitando de un trabajo informal a otro y sin un lugar fijo donde vivir junto con su hijo. Estas trayectorias, a modo de ejemplo de otras tantas reconstruidas a lo largo del trabajo de campo, ilustran tres cuestiones centrales a tener en cuenta: en primer lugar, el hecho de que las mujeres migrantes peruanas encuentren en el trabajo doméstico remunerado su primera - y a veces única - inserción laboral en Argentina no se relaciona directamente con la pertenencia de clase ni con la calificación laboral; en segundo lugar, las escasas opciones laborales para las - y los - migrantes, independientemente de su calificación, muestran la jerarquización y segmentación en términos étnico-nacionales y de género del mercado de trabajo así 
${ }^{65}$ Uno de los trabajos pioneros que ha estudiado esa segmentaciónjerarquización es el de Michael PIORE, 1975. como la precariedad de esas opciones; en tercer lugar, el análisis de las trayectorias de las trabajadoras domésticas migrantes expresa cómo en ciertos contextos socio-históricos algunas clasificaciones sociales se tornan determinantes en relación con las posiciones de los sujetos y las lógicas de explotación y resistencia que se desprenden de esas posiciones.

La interseccionalidad, precisamente, permite reflexionar sobre estas cuestiones, poniendo en el centro de la discusión al mercado de trabajo y su segmentaciónjerarquización entre nativos y migrantes y también entre los/ as propios/as migrantes (y nativos/as). ${ }^{65}$ En tal sentido, brinda herramientas para comprender, en este caso en particular, las diferentes 'formas de ser' trabajadora doméstica migrante, atendiendo a las especificidades de género, clase social, origen étnico-nacional y condición migratoria. Uno de los postulados claves de la interseccionalidad es la disputa de cualquier forma de universalidad que involucre las experiencias de las personas y las estructuras como una totalidad. Esta premisa bien vale para pensar y cuestionar las visiones homogéneas que recaen sobre los/as migrantes. Justamente, esta perspectiva teórico-metodológica pone de relieve que en el marco de una inserción laboral, como el trabajo doméstico remunerado, determinadas clasificaciones se intersectan configurando distintas posibilidades y modalidades de inserción laboral, movilidades verticales y horizontales, y acceso a derechos en tanto trabajadoras.

\section{A modo de cierre}

A lo largo del artículo se explicitaron las principales potencialidades de la interseccionalidad para pensar problemáticas sociales, en particular las migraciones internacionales. La interseccionalidad, de modo fundamental, vino a poner en discusión formas de opresión, falsos universalismos, múltiples clasificaciones sociales y su incidencia en la vida de las personas. El foco puesto en las relaciones de poder y las desigualdades sociales a partir de identificar interseccionalmente distintas clasificaciones sociales, históricamente situadas, ha permitido revelar lógicas de explotación antes invisibilizadas y también las resistencias y estrategias que las personas despliegan en pos de enfrentarlas.

Sin embargo, esta perspectiva presenta algunos claroscuros producto de la complejidad que supone pensar la intersección en términos teóricos y empíricos. No obstante ello, estos 'grises' pueden constituirse también en su principal riqueza, en especial vinculada al hecho de que está 'todo por hacerse'. En tal sentido, las potencialidades de la 
interseccionalidad se encuentran en el campo, en las preguntas de investigación y en el trabajo empírico que busca responder y problematizar esas preguntas. Las indagaciones en torno al modo en que se intersectan las categorías sociales, cuáles se intersectan en un momento histórico concreto y lo que hace esa intersección en la vida de las personas y en las estructuras sociales, plantean importantes retos para los y las investigadoras, tanto en el plano teórico como metodológico.

En las investigaciones sobre migraciones internacionales, específicamente, la interseccionalidad no ha superado todavía la etapa de enunciación. Los estudios sobre migraciones y trabajo, en particular, tienen por delante la tarea de explicar en profundidad cómo la intersección de clasificaciones sociales produce y reproduce formas de explotación laboral que se traducen en desigualdades sociales. A la vez, asumen el desafío de discutir empíricamente los mecanismos de generización, etnización y jerarquización de la fuerza laboral entre migrantes y nativos y entre los propios migrantes para poder identificar, por un lado, los sentidos e implicancias de la segmentación del mercado de trabajo (estructura interseccional) y, por el otro, la reproducción de lógicas de exclusión y las estrategias de resistencias y agenciamiento que despliegan los propios sujetos (identidades interseccionales). En este ámbito, las potencialidades y estímulos de la interseccionalidad activan la apertura de líneas de investigación poco exploradas y desarrolladas aún, las cuales demandan una serie de esfuerzos teórico-metodológicos. Son justamente estos esfuerzos los que pueden redundar en una renovación, siempre bienvenida, de este campo de estudio.

\section{Referências}

ACKER, Joan. "Gendered Institutions: From Sex Roles to Gendered Institutions". Contemporary Sociology, v. 21, n. 5, p. 565-569, sep. 1992.

ANDERSON, Cynthia. "Understanding the Inequality Problematic: from Scholarly Rhetoric to Theoretical Reconstruction". Gender and Society, v. 10, n. 6, p. 729746, dic. 1996.

ANTHIAS, Floya. "Rethinking social divisions: some notes towards a theoretical framework". Sociological Review, v. 46, n. 3, p. 505-553, ago. 1998.

ANTHIAS, Floya. "Género, etnicidad, clase y migración: interseccionalidad y pertenencia translocalizacional". In: RODRIGUEZ, Pilar. Feminismos periféricos. Granada: Editorial Alhulia, 2006, p. 49-68. 
ANTHIAS, Floya. "Transnational Mobility, migration research and intersectionality". Nordic Journal of Migration Studies, n. 2, p. 102-110, jun. 2012.

ANTHIAS, Floya y YUVAL-DAVIS, Nira. "Contextualizing feminism. Gender, ethnic and class divisions". Feminist Review, n. 15, p. 62-74, nov. 1983.

BARRIOS, Luiza. "Nossos Feminismos Revisitados". Revista Estudos Feministas, v. 3, n. 2, p. 458-463, jul. 1995.

BIDASECA, Karina. Perturbando el texto colonial. Los estudios (pos) coloniales en América Latina. Buenos Aires: Editorial $\mathrm{SB}, 2010$.

BRAH, Avtar y PHOENIX, Ann. "Ain't I A Woman: Revisiting Intersecionality". Journal of International Women's Studies, v. 5, n. 3, p. 75-86, may. 2004.

BROWNE, Irene y MISRA, Joya. "The Intersection of Gender and Race in the Labor Market". Annual Review of Sociology, v. 29, p. 487-513, ago. 2003.

BÜRKNER, Hans-Joachim. "Intersectionality: How Gender Studies Might Inspire the Analysis of Social Inequality among Migrants". Population, Space and Place, v. 18, p. 181-195, nov./dic. 2012.

CERRUTTI, Marcela. "La migración peruana a la Ciudad de Buenos Aires: su evolución y características". Población de Buenos Aires, v. 2, n. 2, p. 7-28, 2005.

CRENSHAW, Kimberly. "Mapping the Margins: Intersectionality, Identity Politics, and Violence against Women of Color". Stanford Law Review, v. 43, n. 6, p. 1241-1297, jul. 1991.

CURIEL, Ochy. "Crítica poscolonial desde las prácticas políticas del feminismo antirracista". Nómadas, n. 26, p. 92-101, abr. 2007.

DAVIS, Kathy, "Intersectionality as buzzword: A sociology of science perspective on what makes a feminist theory succesful". Feminist Theory, v. 9, n. 1, p. 67-85, abr. 2008.

EZQUERRA SAMPER, Sandra. "Hacia un análisis interseccional de la regulación de las migraciones. La convergencia de género, raza y clase social". In: SANTAMARÍA, Enrique. Retos epistemológicos de las migraciones transnacionales. Barcelona: Anthropos, 2008, p. 237-260.

FALCÓN AYBAR, María del Carmen y BOLOGNA, Eduardo. "Migrantes antiguos y recientes: una perspectiva comparada de la migración peruana a Córdoba, Argentina". Migraciones Internacionales, v.7, n. 1, p. 235266, ene./jun. 2013.

GARCÍA CASTRO, Mary. "Gênero e poder. Leituras transculturais - quando o sertão é mar, mas o olhar estranha, encalha em recifes". Cadernos Pagu, n. 16, p.49-77, 2001.

GONZALEZ, Lélia. "Racismo e sexismo na cultura brasileira". Ciências Sociais Hoje, n. 2, p. 223-244, 1984. 
GONZALEZ, Lélia. "Por un feminismo afrolatinoamericano". Revista Isis International, v. IX, p. 133-141, jun. 1988.

GROSFOGUEL, Ramón. "Implicaciones de las alteridades epistémicas en la redefinición del capitalismo global: transmodernidad, pensamiento fronterizo y colonialidad global". In: ZULETA, Mónica; CUBIDES, Humberto y ESCOBAR, Manuel. ¿Uno solo o varios mundos? Diferencia, subjetividad y conocimientos en las ciencias sociales contemporáneas. Bogotá: Siglo del Hombre Editores, 2007, p. 99-118.

HERRERA, Gioconda. "Género y migración internacional en el experiencia latinoamericana. De la visibilidad del campo a una presencia selectiva". Política y sociedad, v. 49 , n. 1, p. 35-46, 2012.

HERRERA, Gioconda. "Lejos de tus pupilas. Familia transnacional, cuidados y desigualdad social en Ecuador. Quito: Flacso, 2013.

HILL COLLINS, Patricia. "Toward a New Vision: Race, Class and Gender as Categories of Analysis and Connection". Race, Sex and Class, n. 1, p. 35-45, jun. 1993.

HONDAGNEU-SOTELO, Pierrette. "La incorporación del género a la migración: 'no solo para feministas ni solo para las familias'. In: ARIZA, Marina y PORTES, Alejandro. El país transnacional: migración mexicana y cambio social a través de la frontera. México: Universidad Nacional Autónoma de México, 2007, p. 423-451.

JABARDO, Mercedes. Feminismos negros. Una antología. Madrid: Traficantes de Sueños, 2012.

KERNER, Ina. "Más allá de la unidimensionalidad: conceptualizando la relación entre el racismo y el sexismo". Signos filosóficos, v. 11, n. 21, p. 187-205, ene./jun. 2009.

LUGONES, María. "Colonialidad y género". Tabula Rasa, n. 9, p. 73-101, jul./dic. 2008.

LUGONES, María. "Multiculturalismo radical y feminismos de mujeres de color". Revista Internacional de Filosofía Política, n. 25, p. 61-75, 2005.

MAGLIANO, María José. "Los significados de vivir 'múltiples presencias': Mujeres bolivianas en Argentina". Revista Migraciones Internacionales, n. 24, p. 165-195, ene./jun. 2013.

MALLIMACl, Ana Inés. "Migraciones y géneros. Formas de narrar los movimientos por parte de migrantes bolivianos/ as en Argentina". Revista Estudos Feministas, v. 19, n. 3, p. 751-775, set./dic. 2011.

MALLIMACI, Ana Inés. "Localizando el sentido de las desigualdades. Inclusiones y exclusiones de los/as bolivianos/as en Ushuaia". In: KARASIK, Gabriela. Migraciones internacionales. Reflexiones y estudios sobre la movilidad territorial contemporánea. Buenos Aires: CICCUS, 2013, p. 87-106. 
MCCALL, Leslie. "The Complexity of Intersectionality". Signs: Journal of Women in Culture, v. 30, n. 3, p. 1771-1800, mar./jun. 2005.

MIGNOLO, Walter. La idea de América Latina. La herida colonial y la opción decolonial. Barcelona: Gedisa, 2007.

MOHANTY, Chandra. "Bajo los ojos de occidente. Academia Feminista y discurso colonial". In: SUÁREZ NAVAZ, Liliana y HERNÁNDEZ, Aída. Descolonizando el Feminismo: Teorías y Prácticas desde los Márgenes. Madrid: Cátedra, 2008, p. 112-161.

NASH, Jennifer. "Re-thinking Intersectionality", Feminist Review, n. 89, p. 2-14, jun. 2008.

PARELLA, Sonia. Mujer inmigrante y trabajadora: la triple discriminación. Barcelona: Anthropos, 2003.

PESSAR, Patricia y MAHLER, Sarah. "Transnational Migration: Bringing Gender" In: International Migration Review, v. 37, n. 3, p. 812-846, sep. 2003.

PIORE, Michael. "Notes for a theory of labor market stratification". In: EDWARDS, Richard; REICH, Michael y GORDON, David. Labor Market Segmentation. Lexington: Health and Co, 1975, p. 125-150.

PISCITELLI, Adriana. "Tránsitos: circulación de brasileñas en el ámbito de la transnacionalización de los mercados sexual y matrimonial". Horizontes Antropológicos, v. 15, n. 31, 101 136, ene./jun. 2009.

PISCITELLI, Adriana."Interseccionalidades, categorías de articulação e experiências de Migrantes brasileiras". Sociedade e Cultura, v.11, n.2, p. 263-274, jul. 2008.

PISCITELLI, Adriana. "Corporalidades em confronto: gênero e nacionalidade no marco da indústria transnacional do sexo". Revista Brasileira de Ciências Sociais, v. 22, n. 64 , p. 17-33, jun. 2007.

PLATERO, Raquel (Lucas). "Marañas con distintos acentos: género y sexualidad en la perspectiva interseccional". Encrucijadas. Revista Crítica de Ciencias Sociales, n. 5, p. 44-52, 2013.

POMBO, María Gabriela. "La organización del trabajo doméstico y de cuidados no remunerados en mujeres migrantes procedentes de Bolivia: posibles lecturas desde el feminismo poscolonial". In: BIDASECA, Karina y VAZQUEZ LABA, Vanesa. Feminismos y poscolonialidad. Buenos Aires: Ediciones Godot, 2011 , p. 247-260.

PHOENIX, Ann y PATTYNAMA, Pamela. "Editorial: Interseccionality". European Journal of Women's Studies, v. 3, n. 3, p. 187-192, ago. 2006.

NEIMAN, Guillermo y QUARANTA, Germán. Los estudios de caso en la investigación sociológica. In: VASILACHIS DE GIALDINO, Irene (Coord.). Estrategias de investigación cualitativa. Barcelona: Gedisa, 2007, p. 231-238.

710 Estudos Feministas, Florianópolis, 23(3): 691-712, setembro-dezembro/2015 
QUIJANO, Aníbal. "Colonialidad del poder, eurocentrismo y América Latina". In: LANDER, Edgardo. La colonialidad del saber: eurocentrismo y ciencias sociales. Perspectivas latinoamericanas. Buenos Aires: CLACSO, 2000, p. 201-246.

RESTREPO, Eduardo y ROJAS, Axel. Inflexión decolonial: fuentes, conceptos y cuestionamientos. Popayán: Editorial Universidad del Cauca, 2010.

ROSAS, Carolina. Implicaciones mutuas entre el género y la migración. Mujeres y varones peruanos arribados a Buenos Aires entre 1990 y 2003. Buenos Aires: Eudeba, 2010.

SAFFIOTI, Heleieth. "Rearticulando gênero e classe social". In: COSTA, Albertina de Oliveira e BRUSCHINI, Cristina. Uma questão de gênero. São Paulo: Fundação Carlos Chagas, 1992. p. 183-215.

SALEM, Sara. "Feminismo islámico, interseccionalidad y decoIonialidad”. Tábula Rasa, n. 21, p. 111-122, jul./dic. 2014.

SHIELDS, Stephanie. "Gender: An intersectionality Perspective". Sex Roles, n. 59, p. 310-311, jul. 2008.

STOLCKE, Verena. "¿Es el sexo para el género lo que la raza para la etnicidad...y la naturaleza para la sociedad?". Política y Cultura, n. 14, p. 25-60, sep./dic. 2000.

STOLCKE, Verena. "La mujer es puro cuento: la cultura del género". Revista Estudos Feministas, v. 12, n. 2, p. 77-105, may./ago. 2004.

TIZZIANI, Ania. "De la movilidad ocupacional a las condiciones de trabajo. Algunas reflexiones en torno a diferentes carreras laborales dentro del servicio doméstico en la ciudad de Buenos Aires". Trabajo y Sociedad, v. 15, n. 17, p. 309-328, jun./set. 2011.

VERLOO, Mieke. "Multiple Inequalities, Intersectionality, and the European Union". European Journal of Women's Studies, n. 13, p. 211-228, ago. 2006.

VIVERO VIGOYA, Mara. "La sexualización de la raza y la racialización de la sexualidad en el contexto latinoamericano actual". Revista Latinoamericana de Estudios de Familia, v. 1, p. 63-81, ene./dic. 2009.

WALSH, Catherine. "Interculturalidad, plurinacionalidad y decolonialidad: las insurgencias político-epistémicas de refundar el Estado". Tabula Rasa, n. 9, p. 131-152, jul./dic. 2008.

YUVAL-DAVIS, Nira. "Intersectionality and Feminist Politics". European Journal of Women's Studies, n. 3, v. 13, p. 193209, ago. 2006.

[Recebido em 25 e outubro de 2013 , reapresentado em 27 de fevereiro de 2015 e aceito para publicação em 17 de março de 2015] 


\section{Intersectionality and Migrations: Potentialities and Challenges}

Abstract: The debates on intersectionality occupy a central place in the theorization about gender and migration. This paper explores the potential of intersectionality to think about social processes and experiences, principally, international migration and migrant experiences. Moreover, it analyses some of the 'problems' this perspective presents. In order to accomplish this purpose, it reconstructs the origins and consolidation of intersectionality, its implications in social sciences in general and migration studies in particular, and its methodological and theoretical ambiguities. Finally, this paper recuperates the contributions of intersectionality to empirically study the relationship between work and migration from outlining labor trajectories in paid domestic work of Peruvian women in Argentina. In methodological terms, this research is based on a qualitative work (participant observation and unstructured interviews) developed since 2011 up to the present in Cordoba city (Argentina) with Peruvian women who have been involved in this labor activity in their migration itineraries.

Key Words: Intersectionality; international migration; social divisions; gender studies; paid domestic work.

712 Estudos Feministas, Florianópolis, 23(3): 691-712, setembro-dezembro/2015 